\title{
Una vía hacia la educación médica orientada a las competencias: Los requisitos globales esenciales mínimos *
}

\author{
M. Roy Schwarz y Andrzej Wojtczak ** \\ International Institute for Medical Education (IIME). New York. USA
}

Los médicos también son miembros de una comunidad global. La globalización creada por las economías interconectadas y un lenguaje global, por la revolución informática y la velocidad de desplazamiento, se manifiesta en todos los ámbitos (el mundo científico, la salud pública, el medio ambiente, la seguridad...) y la medicina no queda al margen de este fenómeno por lo que no puede obviarse la búsqueda de una respuesta a la pregunta: “¿Qué clase de médico precisa este mundo globalizado?".

La medicina, sin embargo, se ha considerado a sí misma siempre como una profesión globalizada puesto que el conocimiento, la investigación y la educación médicas siempre han cruzado libremente las fronteras nacionales. Muchas aptitudes de los médicos son universales así como los compromisos de la profesión con los pacientes que no varían con el lugar en que se produce el encuentro médicopaciente (Schwarz 2001).

La libertad de movimientos de los ciudadanos que va en aumento también afecta a los médicos que cada vez en mayor número cruzan las fronteras internacionales, lo que conlleva la necesidad de considerar las diferencias entre los programas formativos en los distintos países así como los procesos y estándares para su cualificación y certificación desde nuevas perspectivas globales. MERCOSUR y la Unión Europea, por ejemplo, ya contemplan la libertad de movimientos a niveles continentales.

\footnotetext{
* Adaptación al castellano de "Medical Teacher 24:125-129, 2002 (www.tandf.co.uk/journals)" por A. Oriol-Bosch, con autorización del IIME

** M. Roy Schwarz, MD es Presidente de la "China Medical Board of New York, USA".

Andrzej Wojtczak, MD es Director del "Instute for International Medical Education”. White Plains, New York, USA.
}

Así los estándares internacionales devienen instrumentos de garantía para las personas pertenecientes a los distintos países. Ya no existen fronteras para las transacciones financieras, telecomunicaciones, transporte aéreo, protección ambiental, la industria farmacéutica ni para la seguridad alimentaria. La educación médica no puede escapar a esta tendencia puesto que lo que los pacientes esperan de sus médicos no puede depender del lugar donde se hayan formado. Todas las facultades de medicina deben producir médicos capaces de responder a las expectativas de los ciudadanos que solicitan sus servicios sean de donde sean.

En los últimos tiempos diversos grupos han hecho recomendaciones para la mejora de la calidad de la educación médica a nivel internacional reconociendo que el entorno está sometido a rápidos cambios globales, sin que por el momento estas recomendaciones hayan recibido un adecuado reconocimiento. Una de estas iniciativas consistió en una reunión que tuvo lugar en Ginebra en 1994 organizada conjuntamente por la OMS y "Educational Comission for Foreign Medical Graduates" bajo el lema "Hacia un consenso global sobre la calidad de la educación médica al servicio de las necesidades individuales y de la población". Expertos de 20 países participantes convinieron que siendo idénticas muchas de las competencias necesarias para todos los médicos estaría justificado establecer estándares globales en la educación médica. Se reconoció que junto a las clásicas competencias nucleares (conocimiento de las bases científicas de la medicina y las habilidades clínicas) existían también otras que no habían sido hasta entonces reconocidas como tales (habilidades relacionales y de comunicación, capacidad de razonamiento y juicio clínico, aptitud para el trabajo en 
equipo, principios y conductas éticas, voluntad autoevaluativa y capacidad para el aprendizaje autodirigido). Este grupo experto predijo la emergencia de un "médico global" en posesión de las competencias necesarias para ejercer en cualquier lugar del mundo y la inevitable necesidad de crear un mecanismo internacional de acreditación para los médicos que garantizara que poseían un núcleo de competencias nucleares universales y también convendría establecer la acreditación internacional de las facultades de medicina. Para alcanzar dichas metas hacia falta definir y consensuar las competencias nucleares de la profesión médica, los currículum nucleares que condujeran a ellas y los instrumentos evaluativos correspondientes para certificarlas. Los cambios ocurridos desde entonces han hecho más necesaria que nunca la definición de lo que significa la profesión médica en una comunidad global.

Posteriormente la Asociación Europea para la Educación Médica tomó un papel activo en esta cuestión y últimamente la Federación Mundial para la Educación Médica puso en marcha un estudio con el fin de desarrollar criterios y estándares que pudieran ser utilizados para la acreditación internacional de las facultades de medicina (WFME 2000) que ha culminado con su presentación en la Conferencia Mundial celebrada en Copenhague el mes de marzo de 2003. Este conjunto de estándares internacionales mayoritariamente se centran en los procesos de la educación médica, ocupándose de la estructura y función de las facultades de medicina, de los procesos educativos, recursos físicos y personal necesarios, etc., tal como es habitual desde hace tiempo en los Estados Unidos y Canadá (US Liasion Committee on Medical Education) para la acreditación de sus facultades.

Aunque sea necesario para una educación de calidad el disponer de políticas de admisión de alumnos adecuadas y de los recursos suficientes, estos elementos por si solos no garantizan que los titulados vayan a adquirir las competencias necesarias para un ejercicio profesional de calidad. Una facultad puede cumplir los requisitos para su acreditación y a pesar de ello no preparar a sus alumnos para que sean competentes en todos los ámbitos considerados esenciales. Ello es posible por cuanto que las competencias esenciales que como mínimo deben exhibir cada uno de sus estudiantes para poder graduarse no han sido definidos ni se han desarrollado los métodos de evaluación de dichas competencias necesarias para determinar si los objetivos competenciales han sido alcanzados.
Con la creciente globalización de la medicina y el concepto emergente de una "profesión médica global”, la cuestión de las competencias esenciales que todos los médicos deben poseer y demostrar en sus conductas profesionales deviene una cuestión central. Su definición ayudaría a los docentes a centrarse en lo que se supone que es esencial, a los estudiantes en lo que se espera que aprendan y a las instituciones qué experiencias educativas debieran ofrecer. Además es obviamente necesario desarrollar los mecanismos que aseguren que todos los graduados de las facultades poseen las competencias exigidas (Schwarz 1998, Hamilton 2000).

En 1999, la "China Medical Board de Nueva York” creó el Instituto para la Educación Médica Internacional (IIME) al que encomendó la responsabilidad de definir las "competencias esenciales mínimas" que todos los licenciados en medicina debieran poseer para poder ser reconocidos como médicos globales (Wojtczak \& Schwarz 2000-2001) de forma que los que posean dichas competencias queden capacitados para seguir una formación postgraduada en una especialidad médica en cualquier lugar.

El proyecto del IIME para dar respuesta a esta mandato se compone de tres fases:

1. La definición de las competencias esenciales mínimas. En esta fase se tenía que:

- Desarrollar los "requisitos globales esenciales mínimos" (RGEM) que definen el conocimiento, las habilidades y las conductas profesionales y éticas que todos los médicos deben exhibir independientemente del lugar donde hubieren adquirido su formación

- Identificar y desarrollar los métodos necesarios para evaluar las competencias de los graduados para así poder evaluar si la facultad está proporcionando las experiencias educativas que permitan a sus estudiantes adquirir dichas competencias

2. Implementación experimental o programa piloto:

- Aplicación de la metodología evaluativa de las competencias para valorar los resultados educativos en un número limitado de facultades en China

- Puesta en práctica de programas correctores de las debilidades en los procesos educativos detectados y su evaluación posterior para comprobar si efectivamente se había logrado corregir las deficiencias

3. Globalización

- Compartir los resultados del proyecto piloto con la comunidad educativa global a fin de mejorar la calidad de la educación médica

- Facilitar el desarrollo de una red global de educación médica 
1a FASE: LA DEFINICIÓN DE LAS COMPETENCIAS ESENCIALES MÍNIMAS

Esta fase comenzó en 1999 con la creación del IIME y la designación de sus tres comités. El primero de ellos, Comité Promotor (Steering Committee) le fue adjudicado un papel de asesor para el liderazgo del Instituto en la implementación del proyecto. El capital de este Comité lo constituía los conocimientos acumulados por los ocho expertos en educación y políticas sanitarias cuya amplia experiencia se había forjado en diferentes países o a nivel internacional. Al segundo grupo denominado Comité Nuclear (Core Committee) se le asignó la tarea de definir y formular los "requisitos globales esenciales mínimos" (RGEM) y se constituyó con 17 expertos en educación médica seleccionados de distintas regiones mundiales. Los "requisitos" debían comprender todos aquellos conocimientos, habilidades, actitudes y conductas que todos los graduados debían necesariamente alcanzar para poder licenciarse, independientemente de cual fuera su facultad de origen. Estos requisitos deberían constituir el núcleo esencial necesario para iniciar su formación postgraduada o para ejercer bajo supervisión. El tercer Comité denominado Comité Consultivo (Advisory Committee) se constituyó con representantes de cada una de las organizaciones mundiales más importantes en el ámbito de la educación mundial. Lo componían los presidentes o sus representantes de 14 organizaciones entre las que se contaban: Accreditation Council for Graduate Medical Education, American Association of Medical Colleges, Association of Medical Education in Europe, American Medical Association, Education Commission for Foreign Medical Graduates, National Board of Medical Examiners, Pan American Federation of the Association of Medical Schools, The Network Community Partnership for Health through Innovative Education (hoy denominada The Network Towards Unity for Health), World Federation for Medical Education, World Health Organization y otras organizaciones nacionales de educación médica. El Comité Consultivo constituye un foro para el intercambio de información y consejo desde las perspectivas de las distintas organizaciones cuyas aportaciones han demostrado ser de un valor incalculable.

La composición de dichos comités puede obtenerse de la web del Instituto www.iime.org/committee/index.htm
El Comité Nuclear del IIME ha definido el núcleo de competencias esenciales mínimas agrupándolas en 7 dominios. Estos dominios fueron identificados en un proceso de revisión bibliográfica o de fuentes inéditas aportadas por los expertos y por agrupación de experiencias y expertezas de los componentes del Comité Nuclear. Los siete dominios identificados por deliberación y consenso y considerados como esenciales son:

- Valores, Actitudes, Conductas y Ética Profesionales

- Bases Científicas de la Medicina

- Habilidades Clínicas

- Habilidades relacionales o comunicativas

- Salud Poblacional y Sistemas Sanitarios

- Gestión de la información

- Pensamiento crítico e Investigación

El significado de cada dominio puede deducirse de los 60 objetivos educativos en que se desglosan y que definen los ámbitos de cada dominio.

La importancia de los dominios "Bases Científicas" y "Habilidades Clínicas" es universalmente aceptada puesto que constituyen los fundamentos de la asistencia médica efectiva. A pesar de la importancia de los otros cinco dominios, hasta ahora no habían sido codificados o definidos como tales de la forma en que lo ha realizado el IIME.

No existe la menor duda en relación que el dominio "Valores, Actitudes, Conductas y Ética Profesionales" refleja las opiniones tanto de la profesión como de la población general y constituye un elemento esencial del ejercicio profesional entendiendo que el ejercicio de la medicina es algo más que un ejercicio de alta complejidad técnica.

La mayoría de las quejas contra los médicos y los servicios asistenciales están relacionados con este dominio. Los médicos deben estar capacitados para afrontar los rápidos avances científicos, incorporar el uso de las nuevas tecnologías de la información y la comunicación, los cambios organizativos y las limitaciones económicas sin perder los valores tradicionales que han dirigido la profesión durante milenios y ser capaces de afrontar los nuevos retos éticos, sociales y legales a los que los médicos deben dar respuesta sin perderlos.

¿Quién puede dudar de la importancia de las habilidades comunicativas como un instrumento esencial de los médicos cuando son tan necesarias para crear un buen clima relacional con los pacientes y sus familiares, o con los otros miembros del equipo de trabajo, los colegas o el público en general?. La buena comunicación es esencial para que el 
médico pueda comprender el contexto de valores, creencias o cultura de los pacientes, en el que deberán aconsejar cuando no educarlos acerca de sus enfermedades, tratamientos, factores de riesgo o estilos de vida saludables.

Haber seleccionado el dominio de "Salud Poblacional y Sistemas Sanitarios" como esencial refleja la creciente convicción en que no basta el conocimiento de las enfermedades y su tratamiento. Dadas las epidemias globales a las que hay que enfrentarse, como el SIDA, el tabaco o la violencia, se precisan los conocimientos y las habilidades para tratar con la salud de las poblaciones, la capacidad para trabajar en equipo con otros profesionales de la salud a fin de promover, mantener e incrementar la salud de la población. Estos esfuerzos deben llevarse a cabo en el contexto de los sistemas sanitarios existentes, motivo por el cual los médicos deben entender como funcionan y bajo que principios se estructuran, así como sus fundamentos legislativos y limitaciones económicas.

Se justifica que la "Gestión de la Información" sea considerada como un dominio de competencias esenciales para los médicos por el hecho de que cada vez más su quehacer dependerá de que disponga de un flujo de información y acceso al conocimiento que ya solo es posible a través de las nuevas tecnologías. Su uso, cada vez más imprescindible, para manejar la información necesaria, para resolver los problemas de salud y para la toma de decisiones en la asistencia a los pacientes. Los médicos necesitan ya y cada vez van a ser más dependientes de su capacidad de utilización de las tecnologías de la información y la comunicación, de la comprensión de sus capacidades y limitaciones en su aplicación a la solución de los problemas médicos y como ayuda para la toma de decisiones.

Finalmente, la consideración del dominio "Pensamiento Crítico e Investigación" como esencial refleja la importancia que tiene para el médico ser capaz de valorar críticamente el conocimiento, la tecnología y la información disponible para resolver los problemas con los que debe enfrentarse. Los médicos deben aplicar los principios de la medicina basada en la evidencia en su toma de decisiones en un entorno de recursos escasos. Deben aprender a valorar críticamente los datos y la información así como comprender el papel de la investigación para la mejora de la calidad del ejercicio profesional, puesto que la medicina de hoy no será la de mañana.

Las grandes transformaciones que debe afrontar el ejercicio de la medicina constituye un enorme reto para los educadores que deben modificar la formación hoy, de forma que prepare a los estudiantes para la medicina de mañana. Es evidente que será necesario que los médicos adquieran de forma continuada nuevos conocimientos y habilidades. Deben adquirir un compromiso consigo mismos para el aprendizaje a lo largo de toda la vida y además han de ser capaces de aprender en esta era de la información. Pero además requieren desarrollar la capacidad emocional e intelectual que les permita ser conscientes y de reconocer sus propias limitaciones, estar dispuestos a autoevaluarse regularmente así como a serlo por sus colegas y permanentemente a dedicar tiempo y esfuerzos al aprendizaje autodirigido.

Estas competencias son vistas como objetivos educativos por la comunidad académica de forma generalizada a juzgar por las publicaciones que están apareciendo en los últimos años.

Los "RGEM" conforman los objetivos de aprendizaje y capacitación globales mínimos para los graduados de las facultades de medicina. Sin embargo es necesario comprender que hace falta atender también las necesidades locales, nacionales o regionales, por tanto es necesario considerar también las peculiaridades culturales y las condiciones socioeconómicas en todo cuanto afecten las relaciones médicopaciente. En consecuencia, un currículum que solo atendiera a los "RGEM" y no lo hiciera a las necesidades propias de su entorno, sería un currículum incompleto. Es necesario comprender que el concepto "RGEM" no implica uniformismo curricular ni de los procesos educativos. Cada facultad debe adoptar su propio diseño curricular aunque asegurando en primer lugar, que se alcanzan las competencias nucleares del "RGEM" y en segundo lugar, todas las necesarias para responder a las peculiaridades locales.

Sin embargo, la aceptación de los "RGEM" y su incorporación en el currículum por si solo no necesariamente producirán los cambios deseados en las competencias de los graduados a no ser que el cambio vaya acompañado de las correspondientes adaptaciones en el proceso evaluador. La evaluación de los productos del aprendizaje expresados en los "RGEM" debe garantizar que la evaluación se centra realmente en los objetivos desde el momento en que se planifica el programa educativo y que los estudiantes, siendo conocedores de cómo van a ser evaluados, estarán adquiriendo dichas competencias antes de que sean normativamente evaluados. Por esta razón, antes de la implementación de este proyecto se constituyó un "Grupo de Trabajo para la Evaluación" compuesto por expertos en tecnologías 
evaluadoras con el fin de desarrollar las estrategias adecuadas para la evaluación de los objetivos educativos contenidos en los "RGEM", puesto que esta tarea imprescindible no estaba disponible en el entorno, muy especialmente la evaluación de los valores y las actitudes profesionales. Para desarrollar los métodos y los procesos necesarios para la evaluación de dichas competencias hará falta desarrollar un programa de investigación que se enriquece con las experiencias disponibles en esta área.

Además, como apoyo al proceso de implementación de los "RGEM" el IIME ha generado un "Glosario de Terminología de la Educación Médica" en el que se definen los términos usados en los documentos del Instituto así como una "Base de datos mundial de Facultades de Medicina" que puede visitarse en la red del Instituto.

\section{$2^{a}$ FASE: IMPLEMENTACIÓN EXPERIMENTAL}

La segunda fase comenzó en 2002 y en ella los "RGEM" se utilizan para evaluar la competencia de los graduados en algunas facultades de medicina chinas, cuidando de que cada facultad los adapte a sus peculiaridades curriculares pero asegurando que se cubran todos los objetivos educativos en todos los dominios. Para la evaluación de las facultades se prevé agrupar los datos de los estudiantes. Después de la evaluación se retroalimentarán los datos obtenidos para la mejora de las deficiencias que sean detectadas, procediéndose a reconocer formalmente los logros alcanzados por las instituciones.

No parece posible valorar adecuadamente cada una de las competencias al primer intento por lo que se contempla como un proceso de mejora continuada en base a la experiencia que se vaya adquiriendo.

\section{3a FASE: GLOBALIZACIÓN}

La fase de diseminación comenzará a la luz de la experiencia obtenida en la segunda fase. Los instrumentos evaluadores, el proceso seguido, los problemas hallados y las áreas de mejora detectadas se compartirán con la comunidad internacional, lo que debería representar un factor positivo para el desarrollo de una red global en educación médica.

\section{CONCLUSIONES OPERATIVAS}

En el proceso en que nos hallamos inmersos ha puesto en evidencia ciertos principios y algunas implicaciones que exponemos:
- Los "RGEM" preparan para el desempeño de los roles que actualmente son necesarios y describen las actitudes, habilidades y conocimientos básicos de un proceso de aprendizaje a lo largo de toda la vida. Los médicos no pueden ser formados como técnicos que cumplen con un recetario de soluciones para problemas definidos porque deben ser profesionales educados capaces de practicar el arte y la ciencia médica en cada momento como corresponde

- El proyecto "RGEM" del IIME es experimental. Nunca ha sido llevado a cabo anteriormente, por la globalidad de sus objetivos y por su enfoque en las competencias terminales exigibles para licenciarse en medicina. A medida que el proyecto va implementándose se aprenderá de la experiencia y aumentará su comprensión y la visión inicial. No es posible confiar en acertar al primer intento y habrá que aceptar la necesidad de introducir ajustes y mejoras con el tiempo.

- Los "RGEM" no son un producto "made in USA" ni los "requisitos" son una imposición a la moda. Son propiedad de todos los que han contribuido e irán contribuyendo a su desarrollo con la esperanza de que algún día lleguen a ser los estándares aceptados por todos en educación médica. De otra forma, si se los contempla como directrices USA para el mundo en desarrollo, los requisitos quedan condenados al fracaso.

- Las competencias definidas en los "RGEM" definen lo que es ser médico y no pretenden definir lo que es ser especialista. Son el núcleo que define la profesión médica.

- Este proyecto es un intento de cooperación con y entre todas las organizaciones activas en educación médica más relevantes del mundo. Con este esfuerzo se pretende reconocer el perfil legítimo y trascendente en el ámbito de la educación médica que dichas organizaciones han tenido y están teniendo actualmente, así como enriquecer el proyecto con la experteza y experiencia que éstas han ido acumulando a lo largo del tiempo. El éxito del proyecto precisa de que todos se lo sientan como suyo.

- No es intención del proyecto que las facultades sean evaluadas por forasteros. La participación en la fase de implementación experimental es voluntaria, los "RGEM" fueron desarrollados por representantes de una aventura global y representan un consenso de un grupo con orientación global. Constituyen el resultado de un esfuerzo de la comunidad global y no emanan de ningún país, organización, ideología o programa en particular

- Algunos programas educativos no cubrirán 
dichos requisitos pero ello no significa que sus productos educativos no sean útiles en los sistemas asistenciales locales, ni tampoco que dichos profesionales no sean médicos de la forma como se define en este proyecto. Por tanto sería conveniente, para referirse a los productos educativos de las facultades con otra denominación que la de la profesión como en ciertas culturas ocurre en algunas profesiones (p.ej. licenciado en derecho vs abogado)

Actualmente muchos líderes del ámbito educativo coinciden en creer que ahora es el momento oportuno para llevar a la práctica un proyecto como este. Si no se utiliza bajo un enfoque constructivo y bajo la tutela del conocimiento y la experiencia de expertos internacionales reconocidos, es posible que aparezcan otros intentos para desarrollar los estándares con aproximaciones menos amistosas. Para la comunidad educativa médica el reto consiste en utilizar la globalización como instrumento y aprovechar la oportunidad para la mejora de calidad y de los resultados educativos (Harden et al. 1999).

Aunque de buen comienzo se intuía que una orientación de la educación médica a las competencias, es decir, a los resultados, debería tener consecuencias importantes sobre los currículum de las facultades, actualmente parece arriesgado predecir cuales serán sus efectos. Creemos que la situación actual tiene analogías con la que existía a principios del siglo XX cuando Abraham Flexner sentó las bases de la educación médica en Norteamérica y Canadá. Los efectos a largo plazo del trabajo de Flexner representaron una notable mejora para la educación médica primero en el continente americano y luego en todo el mundo y con ello una mejor asistencia a los pacientes. Es posible que el esfuerzo del IIME con el proyecto "RGEM" tenga efectos positivos, primero en China seguidos luego por una mejora en el resto del mundo.

\section{BIBLIOGRAFÍA}

HAMILTON, J.D. (2000) Editorial: International standards of medical education: a global responsibility, Medical Teacher, 22 (6), pp.547-548.

HARDEN, R.M., CROSBY, J.R. \& DAVIS, M. H. (1999) AMEE Education Guide No. 14: Outcome-based education, Part 1: An introduction to outcome-based education, Medical Teacher, 21(1), pp.7-14.

SCHWARZ, M.R. (1998) On moving towards international standards in health professions education, Changing Medical Education and Medical Practice [World Health Organization, Geneva].

SCHWARZ, M.R. (2001) Editorial: Globalization and medical education, Medical Teacher, 23(6).

WOJTCZAK A. \& SCHWARZ, M.R. (2000) Minimum essential requirements and standards in medical education, Medical Teacher, 22(6), pp. 555-559.

WOJTCZAK A. \& SCHWARZ, M.R. (2001) International standards in medical education: what they are, and do we need them?, paper presented at the AMEE Conference, 2-5 September, Berlin, Germany.

WORLD FEDERATION FOR MEDICAL EDUCATION TASK FORCE (2000) Defining international standards in basic medical education. Report of a Working Party, Copenhagen 1999, Medical Education, 34(8), pp. 665-675.

WORLD HEALTH ORGANIZATION/EDUCATION COMMISSION FOR FOREIGN MEDICAL GRADUATES (1995) Towards a global consensus on quality medical education: serving the needs of population and individuals, Proceedings of the 1994 WHO/ ECFMG Consultation in Geneva, Switzerland, Academic Medicine, 70(7), Suppl. 